\title{
High-dimensional knots corresponding to the fractional Fibonacci groups
}

\author{
by
}

Andrzej Szczepański (Gdańsk) and Andrĕ̌ Ves n in (Novosibirsk)

\begin{abstract}
We prove that the natural HNN-extensions of the fractional Fibonacci groups are the fundamental groups of high-dimensional knot complements. We also give some characterization and interpretation of these knots. In particular we show that some of them are 2 -knots.
\end{abstract}

1. Introduction. The fractional Fibonacci groups $F^{k / l}(2, m)$ were introduced in [6] for integers $k, l, m$ such that $m \geq 3$ and $(k, l)=1$ :

$$
F^{k / l}(2, m)=\left\langle a_{1}, \ldots, a_{m} \mid a_{i}^{l} a_{i+1}^{k}=a_{i+2}^{l}, i=1, \ldots, m\right\rangle
$$

with all subscripts reduced modulo $m$. In the case $k=l=1$ we get the well-known Fibonacci groups $F(2, m)$ introduced by J. Conway in 1965 . The topological and geometric properties of three-dimensional closed orientable manifolds uniformized by the groups $F(2,2 n)$ and $F^{k / l}(2,2 n)$ were studied in [2] and [6], respectively. Almost all of these groups (and manifolds) are hyperbolic. Concerning the case of $m$ odd, H. Helling pointed out to us that the groups $F(2, m)$ have torsion, and it was shown in [7] that they cannot be fundamental groups of hyperbolic 3-manifolds or hyperbolic 3-orbifolds.

The investigation of HNN-extensions of the Fibonacci groups as highdimensional knot groups was started by J. Hillman in [3], where he considered the natural extension of the group $F(2,6)$ that is the fundamental group of the Euclidean Hantzsche-Wendt manifold. This study was continued in [11], where it was proven that the same HNN-extensions of the

1991 Mathematics Subject Classification: Primary 19D35.

A. Szczepański supported in part by University of Gdańsk (grant number BW 51005-0271-7).

Andrei Vesnin supported in part by Russian Foundation for Basic Research (grant number 98-01-00699).

Both authors are grateful to Banach Center at Warsaw and SFB-343 at Bielefeld University where the research was done. 
Fibonacci groups $F(2, m)$ also arise as high-dimensional knot groups. In the present paper we demonstrate that natural $\mathrm{HNN}$-extensions of the fractional Fibonacci groups $F^{k / l}(2, m)$ are high-dimensional knot groups if and only if $k=1$.

We recall that a group $G$ is said to be an $n$-dimensional knot group, for $n \geq 1$, if $G=\pi_{1}\left(S^{n+2} \backslash K\left(S^{n}\right)\right)$ for some $n$-dimensional knot $K$ : $S^{n} \rightarrow S^{n+2}$. Kervaire [10, Section 11D] obtained the following algebraic characterization of such groups: $A$ group $G$ is a 3-knot group (and so, an $n$-knot group for any $n \geq 3$ ) if and only if it is finitely presentable, $H_{1}(G, \mathbb{Z})$ $=\mathbb{Z}, H_{2}(G, \mathbb{Z})=0$ and $G$ has weight 1 (that is, $G$ is a normal closure of some single element). In dimensions $n=1,2$ the above conditions are necessary but no longer sufficient. Our study of the group extensions of $F^{k / l}(2, m)$ will be based on this criterion and on Zeeman's twist spun construction of knots [12].

2. The natural HNN-extension. The group $F^{k / l}(2, m)$ with the presentation (1) is an example of a cyclically presented group in sense of [5]. We recall that a group $G$ is cyclically presented if for some $n$ and $w$ it has a presentation

$$
G=\left\langle x_{1}, \ldots, x_{m} \mid w, \eta(w), \ldots \eta^{m-1}(w)\right\rangle,
$$

where $\eta: \mathbb{F}_{m} \rightarrow \mathbb{F}_{m}$ is an automorphism of the free group $\mathbb{F}_{m}=\left\langle x_{1}, \ldots, x_{m}\right\rangle$ of rank $m$ given by $\eta\left(x_{i}\right)=x_{i+1}, i=1, \ldots, m$, and $w \in \mathbb{F}_{m}$ is a cyclically reduced word. The fractional Fibonacci groups with the presentation (1) arise in this construction for $w=x_{1}^{l} x_{2}^{k} x_{3}^{-l}$.

Obviously, $\eta$ induces an automorphism $\Phi: G \rightarrow G$ given by $\Phi\left(x_{i}\right)=x_{i+1}$, $i=1, \ldots, m$. Let us define the natural $H N N$-extension $\mathcal{G}$ of a cyclically presented group $G$ :

$$
\mathcal{G}=\left\{G, t \mid t g t^{-1}=\Phi(g), g \in G\right\} .
$$

Thus for the group $F^{k / l}(2, m)$ with the presentation (1) we consider an automorphism $\Phi: F^{k / l}(2, m) \rightarrow F^{k / l}(2, m)$ given by $\Phi\left(a_{i}\right)=a_{i+1}, i=$ $1, \ldots, m$, and the natural $\mathrm{HNN}$-extension

$$
\mathcal{F}_{m}^{k / l}=\left\{F^{k / l}(2, m), t \mid t f t^{-1}=\Phi(f), f \in F^{k / l}(2, m)\right\}
$$

which is defined for the same $k, l, m$ as the group $F^{k / l}(2, m)$ was. We have

THEOREM. The group $\mathcal{F}_{m}^{k / l}$ is a 3 -knot group if and only if $k=1$.

P r o of. We check the conditions of Kervaire's criterion.

(1) First we prove that $\Phi$ is a meridional automorphism if and only if $k=1$; in other words, that the normal closure $A^{k / l}(2, m)$ in $F^{k / l}(2, m)$ of $\left\{f^{-1} \Phi(f) \mid f \in F^{k / l}(2, m)\right\}$ is $F^{k / l}(2, m)$ only for $k=1$ (see [3, p. 123]). In 
fact, there is an obvious epimorphism

$$
h: F^{k / l}(2, m) \rightarrow \mathbb{Z}_{k}=\left\langle\gamma \mid \gamma^{k}=1\right\rangle
$$

given by $h\left(a_{i}\right)=\gamma$ for all $i$. This epimorphism induces an epimorphism of the abelianization:

$$
h^{\mathrm{ab}}: F^{k / l}(2, m)^{\mathrm{ab}} \rightarrow \mathbb{Z}_{k} .
$$

Thus $H_{1}\left(\mathcal{F}_{m}^{k / l}\right)=\mathbb{Z}$ if and only if $k=1$. So, from now on we assume that $k=1$ in our considerations.

(2) We show that $\mathcal{F}_{m}^{1 / l}$ is a normal closure of the element $b=t^{-1} a_{1}$. In fact,

$$
t^{-1} a_{2}=t^{-1}\left(t a_{1} t^{-1}\right)=a_{1} t^{-1}=t\left(t^{-1} a_{1}\right) t^{-1}=t b t^{-1},
$$

and for $i=1, \ldots, m$ we get

$$
\begin{aligned}
t^{-1} a_{i} & =t^{-1}\left(t^{i-1} a_{1} t^{-(i-1)}\right)=t^{i-2} a_{1} t^{-(i-1)} \\
& =t^{i-1}\left(t^{-1} a_{1}\right) t^{-(i-1)}=t^{i-1} b t^{-(i-1)} .
\end{aligned}
$$

Let $\mathcal{B}$ be the normal closure of $b$ in $\mathcal{F}_{m}^{1 / l}$. Consider the canonical projection

$$
\varrho: \mathcal{F}_{m}^{1 / l} \rightarrow \mathcal{F}_{m}^{1 / l} / \mathcal{B}
$$

and write $\varrho(a)=\bar{a}$ for $a \in \mathcal{F}_{m}^{1 / l}$. Then $\bar{a}_{1}=\ldots=\bar{a}_{m}=\bar{t}$. For any $i$ we get $\bar{a}_{i}^{l} \bar{a}_{i+1}=\bar{a}_{i+2}^{l}$, hence $\bar{t}=e$ is the neutral element. Therefore $\bar{a}_{1}=\ldots=$ $\bar{a}_{m}=\bar{t}=e$ and $\mathcal{F}_{m}^{1 / l}=\mathcal{B}$. Thus $\mathcal{F}_{m}^{1 / l}$ has weight 1 .

(3) From the short exact sequence of groups

$$
1 \rightarrow F^{k / l}(2, m) \rightarrow \mathcal{F}_{m}^{k / l} \rightarrow \mathbb{Z} \rightarrow 1
$$

we have the Hochschild-Serre spectral sequence

$$
E_{p, q}^{2}=H_{p}\left(\mathbb{Z}, H_{q}\left(F^{k / l}(2, m)\right)\right)
$$

[1, p. 171]. So, it is enough to prove that

$$
\begin{aligned}
& E_{2,0}^{2}=H_{2}\left(\mathbb{Z}, H_{0}\left(F^{k / l}(2, m)\right)\right)=0, \\
& E_{1,1}^{2}=H_{1}\left(\mathbb{Z}, H_{1}\left(F^{k / l}(2, m)\right)\right)=H_{1}\left(F^{k / l}(2, m)\right)_{\mathbb{Z}}=0, \\
& E_{0,2}^{2}=H_{0}\left(\mathbb{Z}, H_{2}(F(2, n))\right)=0 .
\end{aligned}
$$

The first equality is obvious. For the proof of the next one we use Hopf's formula to get a 5 -term exact sequence [1, p. 47]

$$
H_{2}\left(\mathcal{F}_{m}^{k / l}\right) \rightarrow H_{2}(\mathbb{Z}) \rightarrow H_{1}\left(F^{k / l}(2, m)\right)_{\mathbb{Z}} \rightarrow H_{1}\left(\mathcal{F}_{m}^{k / l}\right) \rightarrow H_{1}(\mathbb{Z}) \rightarrow 0,
$$

where the $\mathbb{Z}$-action on $H_{1}\left(F^{k / l}(2, m)\right)$ is induced by the conjugation action of $\mathcal{F}_{m}^{k / l}$ on $F^{k / l}(2, m)$. We have $H_{2}(\mathbb{Z})=0, H_{1}(\mathbb{Z})=\mathbb{Z}$. Suppose $k=1$; then by (1), $H_{1}\left(\mathcal{F}_{m}^{1 / l}\right)=\mathbb{Z}$. Thus 


$$
0 \rightarrow H_{1}\left(F^{1 / l}(2, m)\right)_{\mathbb{Z}} \rightarrow \mathbb{Z} \rightarrow \mathbb{Z} \rightarrow 0,
$$

and so $H_{1}\left(F^{1 / l}(2, m)\right)_{\mathbb{Z}}=0$.

The abelianization $F^{k / l}(2, m)^{\mathrm{ab}}$ has the following property.

Lemma. For any $k, l, m$ the group $F^{k / l}(2, m)^{\text {ab }}$ is finite of order

$$
\left|F^{k / l}(2, m)^{\mathrm{ab}}\right|=d_{m}^{k / l}= \begin{cases}c_{m}^{k / l}+l^{2} c_{m}^{k / l}, & m \text { odd }, \\ c_{m}^{k / l}+l^{2} c_{m-2}^{k / l}-2 l^{m}, & m \text { even },\end{cases}
$$

where

$$
c_{m}^{k / l}=k c_{m-1}^{k / l}+l^{2} c_{m-2}^{k / l},
$$

with $c_{0}^{k / l}=1$ and $c_{1}^{k / l}=k$.

Pr o of. The lemma is obvious by direct computation of the determinant of the exponential sum matrix [8].

In particular, from (5) and (6) we get

$$
d_{5}^{k / l}=k^{5}+5 k^{3} l^{2}+5 k l^{4}, \quad d_{6}^{k / l}=k^{6}+6 k^{4} l^{2}+9 k^{2} l^{4} .
$$

Returning to the proof of the theorem, we note that $H_{2}\left(F^{k / l}(2, m)\right)=0$. In fact, from Hopf's formula [1, p. 46] the number of generators of the group $H_{2}\left(F^{k / l}(2, m)\right)$ is $r-g+w$. Here $g$ is the number of generators and $r$ the number of relations of the group $F^{k / l}(2, m)$, and $w=\operatorname{rank}\left(H_{1}\left(F^{k / l}(2, m)\right)\right)$. In our case $g=r=m$, and by the lemma above, $w=0$. So, $H_{2}\left(F^{k / l}(2, m)\right)$ $=0$ and $E_{0,2}^{2}=0$. Summing up we have shown that $H_{2}\left(\mathcal{F}_{m}^{1 / l}\right)=0$.

3. The fibred 2-knots. As we remarked in the introduction the Kervaire conditions of the high-dimensional knot groups are also necessary when $n=1$ or 2 , but are then no longer sufficient. However, it is proven in [4, p. 34] that if a group $G$ is a torsion free 3-knot group such that $G^{\prime}$ is the fundamental group of a closed orientable 3-manifold $M$ whose factors are Haken, hyperbolic or Seifert fibred, then $G$ is the group of a fibred 2-knot with closed fibre $M$.

We recall [6] that the fractional Fibonacci group $F^{1 / l}(2,2 n), l \geq 1, n \geq 3$, is the fundamental group of a closed orientable 3-manifold $\mathcal{M}_{n}^{1 / l}$, which can be obtained as an $n$-fold cyclic covering of the two-bridge knot $\left(2 l+\frac{1}{2 l}\right)$. The manifolds $\mathcal{M}_{n}^{1 / l}$ are hyperbolic with one exceptional case $\mathcal{M}_{3}^{1}$, which is the Euclidean Hantzsche-Wendt manifold that is Seifert fibred. Thus we have the following corollary of the above theorem.

Proposition 1. For $l \geq 1$ and $n \geq 3$ the group $\mathcal{F}_{2 n}^{1 / l}$ is a fibred 2 -knot group with closed fibre $\mathcal{M}_{n}^{1 / l}$. 
Recall that by the definition (4) the automorphism $\Phi$ corresponding to the group $\mathcal{F}_{2 n}^{1 / l}$ is of order $2 n$.

We now show that there are other HNN-extensions of the group $F^{1 / l}(2,2 n)$. Indeed, the $n$-fold cyclic covering $\mathcal{M}_{n}^{1 / l}$ of the 2-bridge knot $\left(2 l+\frac{1}{2 l}\right)$ is induced by the automorphism $\Psi: F^{1 / l}(2,2 n) \rightarrow F^{1 / l}(2,2 n)$ given by $\Psi\left(a_{i}\right)=a_{i+2}, i=1, \ldots, 2 n$.

Define the HNN-extension

$$
\mathcal{G}_{n}^{l, p}=\left\{F^{1 / l}(2,2 n), t \mid t f t^{-1}=\Psi^{p}(f), f \in F^{1 / l}(2,2 n)\right\} .
$$

By a general construction of a twist spun knot ([9], [10, Section 3L]) we see that for $0<p<n,(p, n)=1$, the group $\mathcal{G}_{n}^{l, p}$ is a fundamental group of a $p$-twist spun of a $\left(2 l+\frac{1}{2 l}\right)$-knot, which can be described as a fibred knot with fibre $\operatorname{punc}\left(\mathcal{M}_{n}^{1 / l}\right)$. We denote this 2 -knot by $\mathcal{K}_{n}^{l, p}$. Here $\operatorname{punc}\left(\mathcal{M}_{n}^{1 / l}\right)$ denotes a manifold $\mathcal{M}_{n}^{1 / l}$ minus an open ball around the branch set. As a corollary of these considerations, we get

Proposition 2. For $n \geq 3, l \geq 2,0<p<n,(p, n)=1$ the group $\mathcal{G}_{n}^{l, p}$ is a fibred 2-knot group with fibre $\operatorname{punc}\left(\mathcal{M}_{n}^{1 / l}\right)$.

For integers $p, q$ satisfying $0<p, q<n,(p, n)=(q, n)=1$ we can distinguish knots $\mathcal{K}_{n}^{l, p}$ and $\mathcal{K}_{n}^{l, q}$ in virtue of [9]. The groups $\mathcal{G}_{n}^{l, p}$ and $\mathcal{G}_{n}^{l, q}$ are isomorphic if and only if $\Psi^{p}$ is conjugate to $\Psi^{ \pm q}$ in $\operatorname{Out}\left(F^{1 / l}(2,2 n)\right)$. The knot exteriors are homeomorphic only if $\Psi^{p}$ is conjugate to $\Psi^{ \pm q}$ in $\operatorname{Aut}\left(F^{1 / l}(2,2 n)\right)$.

We remark that the extension (7) can also be regarded as a natural HNNextension of a cyclically presented group. Indeed, if we suppose $y_{i}=a_{2 i}$ for $i=1, \ldots, n$, then $a_{2 i+1}=y_{i}^{-l} y_{i+2}^{l}$. Therefore we get the following cyclic presentation for the fractional Fibonacci group:

(8) $F^{1 / l}(2,2 n)=\left\langle y_{1}, \ldots, y_{n} \mid\left(y_{i}^{-l} y_{i+1}^{l}\right)^{l} y_{i+1}=\left(y_{i+1}^{-l} y_{i+2}^{l}\right)^{l}, i=1, \ldots, n\right\rangle$, and the automorphism $\Psi\left(y_{i}\right)=y_{i+1}$. Thus the group $\mathcal{G}_{n}^{l, 1}$ is the natural HNN-extension of the cyclically presented group (8).

\section{References}

[1] K. Brown, Cohomology of Groups, Springer, New York, 1982.

[2] H. Helling, A. Kim and J. Mennicke, A geometric study of Fibonacci groups, J. Lie Theory 8 (1998), 1-23.

[3] J. Hillman, Abelian normal subgroups of two-knot groups, Comment. Math. Helv. 61 (1986), 122-148.

[4] -, 2-Knots and Their Groups, Austral. Math. Soc. Lecture Ser. 5, 1989.

[5] D. Johnson, Presentation of Groups, London Math. Soc. Lecture Note Ser. 22, Cambridge Univ. Press, 1976. 
[6] A. Kim and A. Vesnin, A topological study of the fractional Fibonacci groups, Siberian Math. J. 39 (1998).

[7] C. MacLachlan, Generalizations of Fibonacci numbers, groups and manifolds, in: Combinatorial and Geometric Group Theory (Edinburgh, 1993), A. J. Duncan, N. D. Gilbert and J. Howie (eds.), London Math. Soc. Lecture Note Ser. 204, Cambridge Univ. Press, 1995, 233-238.

[8] W. Magnus, A. Karras and D. Solitar, Combinatorial Group Theory, Wiley Interscience, New York, 1966.

[9] S. Plotnik, Equivariant intersection forms, knots in $S^{4}$, and rotations in 2-spheres, Trans. Amer. Math. Soc. 296 (1986), 543-575.

[10] D. Rolfsen, Knots and Links, Publish or Perish, Berkeley, CA, 1976.

[11] A. Szczepański, High dimensional knot groups and HNN extensions of the Fibonacci groups, J. Knot Theory Ramifications 7 (1998), 503-508.

[12] C. Zeeman, Twisting spun knots, Trans. Amer. Math. Soc. 115 (1965), 471-495.

Institute of Mathematics

University of Gdańsk

Wita Stwosza 57

80-952 Gdańsk, Poland

E-mail: matas@paula.univ.gda.pl
Sobolev Institute of Mathematics Siberian Branch of Russian Academy of Sciences

Novosibirsk, 630090, Russia

E-mail:vesnin@math.nsc.ru

Received 21 October 1997; in revised form 10 February 1998 